\title{
Komposition (oder Halbaffigierung) zum Ausdruck von Nominalaspekt: Schmuckstück, Glücksfall und Zuckerwerk
}

\author{
Gisela Zifonun
}

\section{Vorstellung der Phänomene und Thesenformulierung}

Gegenstand des vorliegenden Beitrags sind Phänomene der folgenden Art:

(1) a. Reiskorn, Grashalm, Goldbarren, Salatkopf

b. Schmuckstück, Gebäckstück, Geldstück, Erbstück

(2) a. Glücksfall, Todesfall, Trauerfall

b. Störfall, Zwischenfall

(3) a. Zuckerwerk, Liedgut, Bettzeug

b. Naschwerk, Grillgut

Es handelt sich um Komposita oder kompositionsähnliche Strukturen mit einem grammatisch interessanten Zweitglied, dem Kopf der Konstruktion. In (1a) werden aus Kontinuativa (mass nouns) Individuativa (count nouns) erzeugt, in (1b) ist insbesondere das Zweitglied -stück für die Individuation zuständig.

In (2) dient das Zweitglied -fall als Bildungsmittel für abstrakte Individuativa auf nominaler (2a) oder anderer Basis (2b) (vgl. Zifonun 2009).

In (3a) werden aus Kontinuativa wie Zucker oder Individuativa wie Lied, Bett Kollektiva erzeugt. In (3b) ist Kollektivbildung auf Verbalstämme wie nasch- oder grill- ausgedehnt. Im Folgenden wird folgende These entwickelt:

Im kompositionsfreudigen Deutschen gibt es Systemansätze für den Ausdruck von ,Nominalaspekt ${ }^{6}$ durch weitgehend semantisch ausgebleichte nominale Zweitglieder (,Köpfe) von Komposita. Dabei werden insbesondere Substantive mit dem Nominalaspekt, (individuatives) Singulativum' und ,kontinuatives Kollektivum' erzeugt.

Mit Blick auf solche Zweitglieder sprechen wir jeweils von ,Singulativ'bzw. ,Kollektivmarker‘. Ob hier der Terminus ,Halbaffix“ bzw. „Affixoid“ angebracht ist, wird erst in Abschnitt 5 gesondert erörtert. 


\section{Seinsart und Nominalaspekt}

Wir schließen hier locker jeweils an Rijkhoffs (2004) Konzept von Seinsart und nominal aspect an. Unter Seinsart - ein Terminus, der parallel zu der Verbalkategorie ,Aktionsart' gebildet ist - versteht Rijkhoff die grundlegenden nominalen Subkategorien, die in den Sprachen der Welt unterschieden werden. Er unterscheidet mithilfe der Merkmale \pm Shape (,having a definite outline or not) und \pm Homogeneity (,being agglomerative or not') die folgenden Seinsarten:

- General noun (-Shape)

- Sort noun (-Shape, -Homogeneity)

- Mass noun (-Shape, +Homogeneity)

- Set noun (+Shape)

- Singular object noun (+Shape, -Homogeneity)

- Collective noun (+Shape, + Homogeneity)

Ebenfalls auf einem Paar vergleichbarer binärer Merkmale, nämlich [ \pm bounded] und [ \pm internal structure], beruhen die ,semantic categories of noun phrases" in Jackendoff (1991), eine Modellierung, die besonders einflussreich war.

In den indoeuropäischen Sprachen, wie etwa im Deutschen, Englischen, Französischen, sind bekanntlich die folgenden beiden Seinsarten vertreten:

- Mass noun (-Shape, +Homogeneity)

- Singular object noun (+Shape, -Homogeneity)

Üblicherweise werden hier jeweils die englischen Bezeichnungen mass noun - count noun gebraucht, während sich in der deutschen Grammatikografie bisher keine einheitliche Terminologie durchsetzen konnte. ${ }^{1}$ Wie ganz dezidiert der Rijkhoffsche Ansatz leiden auch solche traditionellen Unterscheidungen m.E. daran, dass sie letztlich außersprachliche Eigenschaften

1 Wie u.a. in Thieroff (2000: 38ff) nachzulesen, umfasst in der grammatischen Tradition ,Appellativum /,Gattungsname“ häufig nicht die Kontinuativa, sondern wird im Sinne von ,Individuativum verwendet. Die Oberklasse für Individuativa und Kontinuativa wird häufig stattdessen durch den Terminus ,Communia“ (,common noun“) bezeichnet oder auch durch die Verlegenheitslösung ,Gattungsnamen im weiteren Sinne` gegenüber ,Gattungsnamen im engeren Sinne' (= Individuativa) (vgl. die Klassifizierung bei Heyse 1838, Duden 1998; beides nach Thieroff a.a.O.). In Duden 2005 umfasst der Terminus ,Appellativum' auch die so genannten ,Stoffbezeichnungen'. Für letztere wiederum werden auch die Termini ,Stoffsubstantiv' (Eisenberg 2004) oder ,Massenomen` verwendet. 
der bezeichneten Entitäten, nach Rijkhoff „spatial properties“ (S. 59) heranziehen, auch wenn immer betont wird, dass nicht eine objektive Wirklichkeit sprachlich abgebildet werde, sondern die Realitätsrepräsentation ihrerseits durch sprachliche Vorgaben gesteuert sei.

Wir ziehen es vor, die Bestimmung der Seinsarten oder basalen nominalen Subkategorien nach deren grammatischem Verhalten vorzunehmen und auch terminologisch einen anderen Weg einzuschlagen. Wir sprechen einerseits von Individuativa, insofern es sich um Substantive handelt, die als Kern von Nominalphrasen mit Individuenreferenz fungieren, andererseits von Kontinuativa, für die dieses referenzsemantische Charakteristikum nicht gilt. Bedeutsamstes grammatisches Merkmal der Individuativa, das direkt ihren referenzsemantischen Status widerspiegelt, ist in der Tat entsprechend der englischen Begrifflichkeit die Verbindbarkeit mit Kardinalzahlen. Grammatisch charakteristisch für die Individuativa der indoeuropäischen Sprachen ist darüber hinaus ihr obligatorischer Numeruskontrast - bei Bezug auf eine Vielheit von Individuen muss der Plural gewählt werden. ${ }^{2}$ Für Kontinuativa gilt die Negation beider Merkmale:

- $\quad$ statt mass noun (-Shape, +Homogeneity):

Kontinuativum [-Numeruskontrast, - Kardinalzahl]

- statt singular object noun (+Shape, -Homogeneity):

Individuativum [+Numeruskontrast, +Kardinalzahl]

Mit diesen Bestimmungen sind die Seinsarten ,Individuativum' und ,Kontinuativum' anwendbar auf den gesamten Substantivwortschatz des Deutschen, während bei ontologisch-lokalistischen Bestimmungen nur die Konkreta direkt erfasst sind. Bei Anwendung auf den Abstraktwortschatz kann allenfalls metaphorisch von Shape/Gestalt die Rede sein. Auch wo es um Substantive geht, denen das begriffliche Verfahren der Kollektion zugrunde liegt und mehrere Individuen begrifflich z.B. zu einer Mannschaft oder Gesindel zusammengefasst werden, bedarf es gewisser Verrenkungen, um hier die Zuweisung von +Shape (bei Mannschaft) bzw. -Shape (bei Gesinde) zu rechtfertigen (vgl. dazu im Einzelnen Abschnitt 4). Wir können insgesamt von folgender Struktur ausgehen, bei der die dominante Unterscheidung zwischen Individuativa und Kontinuativa mit

2 Das gilt z.B. für das Ungarische nicht. Dort wird in bestimmten Kontexten auch bei Bezug auf Vielheiten der Singular gesetzt. In Verbindung mit Kardinalzahlen und anderen Quantifikatoren ist Singularsetzung obligatorisch. Wir ziehen hier - trotz merkmalslogischer Redundanz - zur Abgrenzung beide Merkmale (also Numeruskontrast und Kardinalzahl) heran, um auch abweichende Fälle zu erfassen, bei denen z.B. Numeruskontrast, aber keine Verbindbarkeit mit Kardinalzahlen vorliegt wie in die Wasser, die Fluten (Abundanzplural). 
der dazu orthogonalen Unterscheidung zwischen Konkreta, Abstrakta und Kollektiva verknüpft wird:

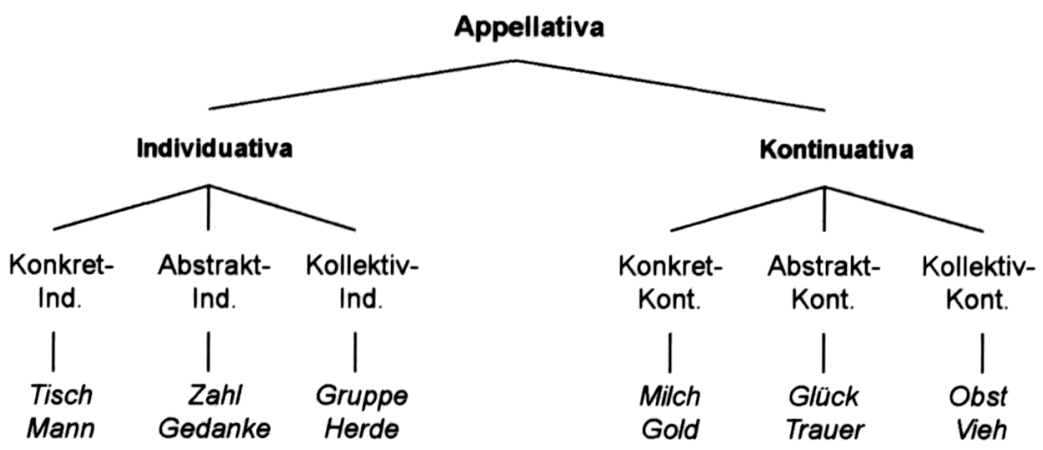

Schema 1: Hierarchie der Seinsarten für das Deutsche ${ }^{3}$

Den Terminus ,Nominalaspekt ${ }^{\star}$ - parallel zu ,Aspekt ${ }^{\star}$ als Verbalkategorie - verwendet Rijkhoff, ${ }^{4}$ wenn Seinsarten offen mit morphologischen Mitteln ausgedrückt werden; er zieht dabei nur flexionsmorphologische Mittel in Betracht.

There are two kinds of nominal aspect markers: singulative aspect markers, which indicate that the set consists of one individual and collective aspect markers, which indicate that the set consists of more individuals. (Rijkhoff 2004: 121)

Die Anwendbarkeit von Nominalaspektmarkern betrifft bei Rijkhoff Sprachen mit „general nouns“ bzw. in anderer Terminologie ,transnumeralen' Nomina. Andererseits scheint in gewisser Weise Vergleichbares auch in indoeuropäischen Sprachen mit ihren anders gelagerten Seinsarten zu geschehen.

3 Im Folgenden wird sowohl von der Bezeichnung, Konkret-Individuativum" usw. Gebrauch gemacht als auch von, individuativem Konkretum/Konkretsubstantiv' usw.

4 Die entsprechende Stelle lautet: „We can define the notion ,aspect' as the way in which a property or relation designated by a predicate is represented in some dimension. Depending on the kind of predicate involved, two kinds of aspect can be distinguished: verbal and nominal aspect. Verbal aspect is concerned with representations in the temporal dimension, and nominal aspect with representations in the spatial dimension". (Rijkhoff 2004: 101) Die Parallele betrifft abstrakte Strukureigenschaften wie vorhandene oder fehlende ,Homogenität bei atelischem Verbalaspekt und bei mass nouns auf der einen Seite und telischen Verben und singular object nouns auf der anderen Seite. Nicht gemeint ist bei diesem allgemeinen Verständnis, dass Nominalaspekt Perspektivierungen in der Zeit betreffe, wie sie bei Nomina, die durch Wortbildungsprozesse mit Verben zusammenhängen, gegeben sein können. Allerdings dürften über zeitliche Perspektivierung definierte Klassen wie etwa ,activity', ,accomplishment' (im Vendlerschen Sinne) mit den Klassen des Nominalaspekts (nach Rijkhoff) systematisch korrelieren. 
So gibt es eine lange Tradition vor allem für die Beschreibung von Singulativa in europäischen Sprachen, insbesondere dem Russischen mit Mittel der Derivationsmorphologie; vgl. Birkenmaier (1980), Drossard (1982), Greenberg (1972), Koptjevskaja-Tamm (2004). Dort erfolgt die Abbildung von kontinuativen Bezeichnungen für Pflanzliches (z.B. Beerensorten, Hülsenfrüchte) auf individuative Bezeichnungen für Einzelexemplare mithilfe der Suffixe -ina, -ica wie in den Paaren:

(4) a. gorox - gorošina ,Erbse

b. fasol' - fasolina ,Bohne'

c. vinograd - vinogradina ,Traube

d. luk - lukovica ,Zwiebel'

Von dort her wurde das Konzept übertragen auf die Verhältnisse in westeuropäischen Sprachen, etwa in Löbel (1986). Dabei legt sie ein enges Verständnis von Singulativum zugrunde:

Unter Singulativum verstehen wir die Bezeichnung der kleinsten natürlichen Einheiten wie Korn, Bobne, Halm von dispersiven Massen wie Reis, Kaffee, Gras. (Löbel 1986: 22)

Löbel beschreibt daneben auch die Rolle der Komposition bei singulativen Bezeichnungen wie in Reiskorn, Kaffeebohne, Grashalm.

$\mathrm{Da}$ auch in Löbels Bestimmung außersprachliche Gesichtspunkte zu dominant erscheinen, fasse ich den Begriff semantisch weiter, beschränke ihn aber auf Wortbildungsprodukte:

Singulativa sind Individuativa, die mit morphologischen Mitteln (Derivation ggf. auch Komposition) aus Kontinuativa hergeleitet werden. Im Deutschen können Köpfe wie -korn, -bohne, -balm, -kopf, -stück als Singulativmarker, Marker des singulativen Nominalaspekts, betrachtet werden. Hier kann verdeutlichend auch von ,singulativen Komposita' gesprochen werden.

Die Singulativmarker sind, wie gezeigt werden wird, mit Ausnahme von -stück und -fall nur ,schwach grammatikalisiert'. Grammatikalisierung verstehen wir hier als eine Form von Sprachwandel, bei der lexikalische Einheiten und Konstruktionen in sprachliche Kontexte eingehen, in denen sie grammatische Funktionen wahrnehmen. ${ }^{5}$ Dabei finden keine abrupten Wechsel statt, sondern eine Serie von subtilen Übergängen, entlang einem so genannten ,Grammatikalisierungspfad“ (auch: „cline“):

Inhaltswort $>$ grammatisches Wort $>$ Klitikon $>$ Flexionsaffix

(vgl. Hopper \& Traugott 2003: 7)

5 Ich schließe hier an die Definition in Hopper \& Traugott (2003: 1) et pass. an. 
Von ,schwach grammatikalisiert' spreche ich, wenn es sich nach wie vor um Inhaltswörter mit ihrer gegebenen lexikalischen Bedeutung handelt, bei denen sich eine grammatische Funktion, hier die des Singulativmarkers, in bestimmten sprachlichen Umgebungen als eine Art Seiteneffekt einstellt.

Im Gegensatz zu Singulativmarkern, deren Existenz in der Wortbildungsmorphologie des Deutschen nicht thematisiert wurde (vgl. aber Wiese 1997: 224), ist das Vorhandensein von Kollektivmarkern wohlbekannt: Es handelt sich um das Präfix Ge- (wie in Getier, Geäst, Gemäuer), die Suffixe -heit (wie in Menschbeit, Christenheit), -schaft (wie in Ärzteschaft, Arbeiterschaft, Dienerschaft) und -tum (wie in Bürgertum, Weltchristentum). Auch die kollektivierende Kraft der Elemente -gut, -werk und -zeug wird in der einschlägigen Literatur gesehen, wobei die Einordnung elementabhängig und abhängig von den Autoren von Suffix über den Status als Halbsuffix/Suffixoid bis zum Kompositumglied reicht (vgl. dazu im Einzelnen Abschnitt 5). Uns kommt es an dieser Stelle auf die Betrachtung als Nominalaspekt-Markierung an und wir bestimmen:

Die Elemente -gut, -werk, -zeug sind Kollektivmarker, Marker des kollektiven Nominalaspekts. Sie leiten aus Erstgliedern nicht-kollektiver Natur kontinuative Kollektiva ab.

Diese Kollektivmarker sind stärker grammatikalisiert als die oben betrachteten Singulativmarker (vgl. dazu Abschnitt 4).

Die Wirkung beider Markersorten besteht in der Erzeugung von Substantiven der Seinsart Individuativum (bei den Singulativmarkern) bzw. kollektivem Kontinuativum (bei den Kollektivmarkern). Bei substantivischer Basis erfolgt dabei eine Abbildung von einer Seinsart bzw. Nominalklasse in eine andere. Hinzu kommen als weitere Erstglieder verbale und adjektivische Stämme sowie Präpositionen. Dies verdeutlichen wir durch die beiden folgenden Schemata: 


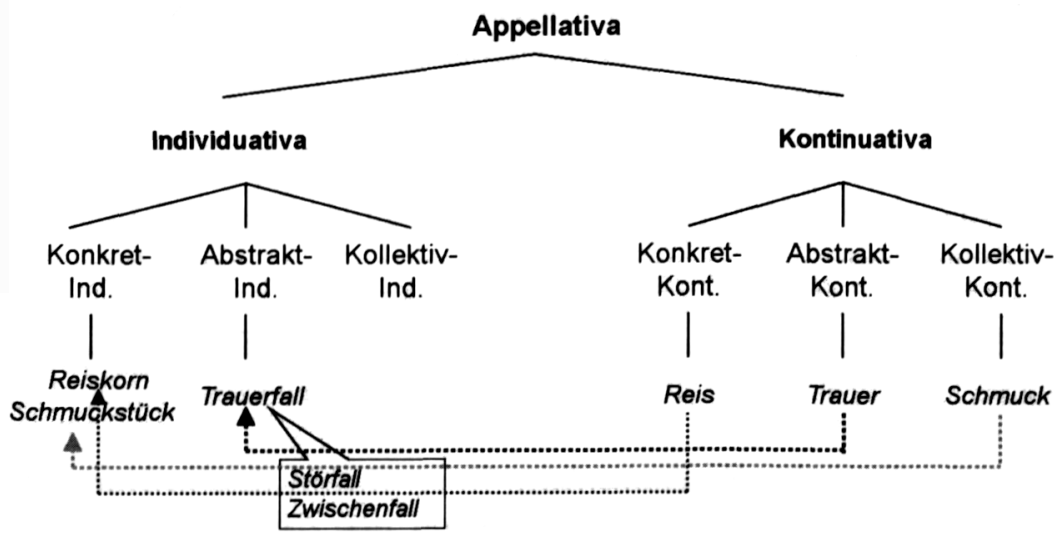

(Schema 2: Singulativa aus Kontinuativa)

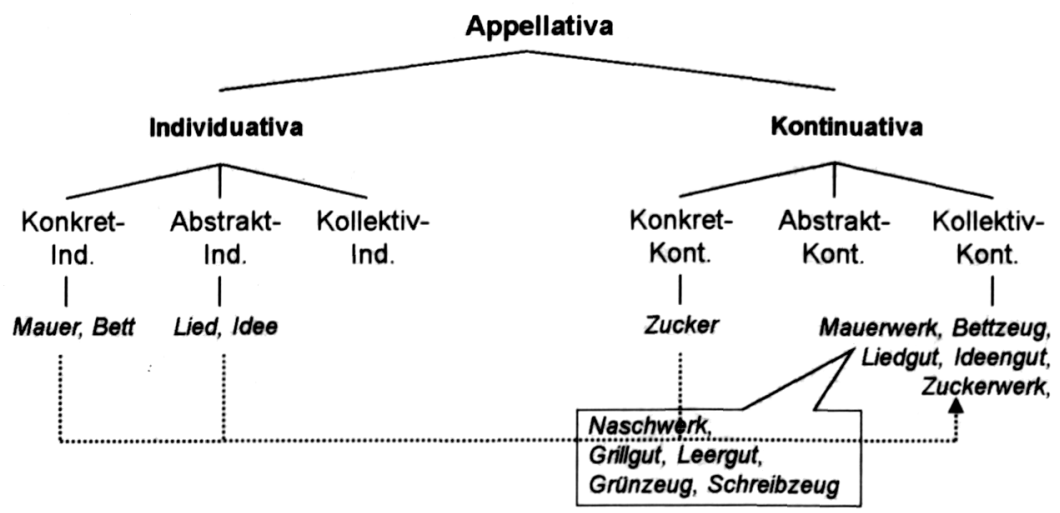

Schema 3: Kontinuative Kollektiva aus nicht-kollektiven Erstgliedern

\section{Singulativa}

Wir betrachten nun zunächst die Singulativa. Das Muster ist im Deutschen nur schwach grammatikalisiert. Die semantisch-kommunikative Motivation für die Bildung von Singulativa ist der Bedarf nach Bezeichnungen für die einzelnen auf natürliche Weise oder durch künstliche Portionierung abgegrenzten Objekte, die unter einen Kontinuativbegriff fallen, und damit auch die Ermöglichung des Zählens und der numeri- 
schen Quantifikation. Eben dieser Bedarf kann aber auch durch Numerativkonstruktionen (vgl. Krifka 1991: 402) gedeckt werden. Diese haben die allgemeine Struktur:

\begin{tabular}{l|l|l|l}
$\begin{array}{c}\text { [Kardinalzahl/ } \\
\text { Quantifikator }\end{array}$ & $\begin{array}{c}\mathrm{N}(\mathrm{G})_{1} \\
\text { (Numerativ- } \\
\text { substantiv) }\end{array}$ & $\left.\mathrm{N}(\mathrm{G})_{2}\right]_{\mathrm{NP}}$ & \\
\hline zwei & Würfel & Zucker & a) Zählkonstruktion \\
\hline zwei & Stück & Vieh & b) Klassifikatorkonstruktion
\end{tabular}

Für den vorliegenden Zusammenhang ist es wichtig, dass in Numerativkonstruktionen die grammatische Funktion der Zählbarmachung oder auch Singulativität konstruktionell kodiert ist. Die Numerativsubstantive selbst können gemäß ihrer lexikalischen Bedeutung gebraucht werden, ohne selbst grammatikalisiert zu sein (wie etwa Würfel, Bobne, Halm usw.).

Von den verschiedenen bei Krifka a.a.O. genannten Subtypen der Numerativkonstruktionen - z.B. Messkonstruktionen (wie zwei Liter Bier), Behälterkonstruktionen (wie zwei Eimer Wasser) - sind hier vor allem die beiden als a) und b) genannten Typen der Zähl- und der Klassifikatorkonstruktionen einschlägig. Sie stellen potentielle Konkurrenzkonstruktionen zu singulativen Komposita dar. Das Zweitglied des Kompositums entspricht dem Numerativsubstantiv der Numerativkonstruktion:

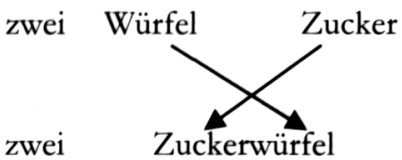

Bei Zählkonstruktionen (in diesem Sinne) ist $\mathrm{N}_{1}$ durch ein „Gestaltnomen“ (vgl. Wiese 1997: 224) vertreten, das die Gestalt des Objekts kennzeichnet. Der Terminus, Klassifikatorkonstruktion' ist von den so genannten Klassifikatorsprachen übernommen, also Sprachen, deren Substantive als „general nouns“ (im Rijkhoffschen Sinne) bzw. als transnumerale Nomina einzuordnen sind, die nicht direkt mit Zahlwörtern verbunden werden können, so dass grundsätzlich zum Zählen von Klassifikatoren (,classifiers') Gebrauch gemacht werden muss. Diese tragen allenfalls sehr allgemeine Merkmale, die die Denotatssorte beschränken, wie etwa \pm belebt, \pm flüssig usw. Im Deutschen wird in erster Linie das Numerativsubstantiv Stück als einem Klassifikator vergleichbar eingestuft, aber auch andere Numerativsubstantive wie Kopf, Sack, Mann in fünf Kopf Salat, zwei Sack 
Zement, fünf Mann Besatzung verhalten sich entsprechend. Klassifikatoren in Numerativkonstruktionen werden nicht in den Plural gesetzt und sind nicht adjektivisch zu NG $\mathrm{NG}_{1}$ erweiterbar, während dies bei Behälter- und Zählkonstruktionen möglich ist (vgl. Wiese 1997: 229ff):

(5) *fünf [große Kopf] Salat - fünf [kleine Körbe] Salat - fünf [große Köpfe] Salat

Hier liegt somit gegenüber anderen Verwendungen von Kopf, Mann, Stück ein Verlust im Hinblick auf typische nominale Eigenschaften vor, nämlich Pluralisierbarkeit und Attribuierbarkeit. Diese Klassifikatoren sind also, anders als die Zählsubstantive, selbst grammatikalisiert.

Wir beschränken uns auf diese beiden Typen von Numerativkonstruktionen, weil nur hier der Vergleich mit entsprechenden Komposita aussagekräftig ist: Bei Messkonstruktionen sind Kompositabildungen ausgeschlossen, vgl. *2pvei Bierliter/Bohnenpfunde; bei Behälterkonstruktionen haben die Komposita eindeutig eine andere Bedeutung als die syntaktische Konstruktion:

(6) a. zwei Eimer Wasser

b. zwei Wassereimer
,Wasser in der Quantität, die von zwei Eimern gefasst werden kann' ,zwei Eimer, die für das Einfüllen von Wasser bestimmt sind ${ }^{6}$

Bei Zählkonstruktionen und konkurrierenden Komposita wie (2wet) Würfel Zucker und (zwel) Zuckerwürfel hingegen liegt kein offensichtlicher Bedeutungsunterschied vor. Allerdings kann es Unterschiede in Frequenz und Usualität geben, daneben möglicherweise auch differierende Figur-GrundVerhältnisse. $^{7}$

Numerativkonstruktionen sind im Deutschen für gewöhnlich appositive Strukturen, in dem Sinne, dass Numerativsubstantiv $\mathrm{N}_{1}$ und das ggf. zur Nominalgruppe $\mathrm{NG}_{2}$ erweiterte Substantiv $\mathrm{N}_{2}$ einfach juxtaponiert

6 Solche Komposita können ihrerseits als Numerativsubstantive gebraucht werden, man vgl. das lexikalisierte Teelöffel, eigentlich ,Löffel zum Abmessen von Tee', das gern als Numerativsubstantiv gebraucht wird: Zwei Teelöffel Salz. Ähnlich könnte auch adhoc formuliert werden: Zwei Wassereimer Bier.

7 Bei den Komposita steht die Objektkonstitution im Vordergrund, bei der Numerativkonstruktion die Quantifikation. Dies zeigt sich z.B. darin, dass qualitative Adjektive der unterschiedlichsten Dimensionen bevorzugt mit dem Kompositum verbunden werden, während in der Numerativkonstruktion überwiegend nur größenbezeichnende Adjektive gebraucht werden: Z.B. (laut COSMAS) verkoblte, verschimmelte, phantasielos präsentierte Brotlaibe gegenüber große/ kleine Laibe Brot. 
sind und $\mathrm{N}(\mathrm{G})_{2}$ im Kasus mit $\mathrm{N}_{1}$ kongruiert oder keine Kasusmarkierung aufweist: mit zwei Flaschen (reinem) Alkohol. Im Grenzfall können solche appositiven Strukturen sich auch als nicht-zählbar erweisen. Darauf kommen wir unten zurück.

Der Vergleich von Numerativkonstruktion bzw. appositiver Konstruktion und singulativem Kompositum kann nun Indizien für den Grad der Grammatikalisierung singulativer Komposita liefern. Dies werden wir im nächsten Abschnitt im Einzelnen zeigen.

\subsection{Numerativkonstruktion versus Kompositum}

Hintergrund ist hier folgende Überlegung: Numerativkonstruktionen bzw. appositive Konstruktionen sind syntaktische Konstruktionstypen, die einerseits auf eine spezifische semantische Interpretation festgelegt sind: Sie realisieren die funktionale Domäne der Quantifikation innerhalb des Nominals (vgl. dazu Zifonun 2010). In diesem Rahmen gibt es andererseits aber bei Zählkonstruktionen (wie auch bei Mess- und Behälterkonstruktionen) ein großes Spektrum der Kombinationsmöglichkeiten von $\mathrm{N}_{1}$ und $\mathrm{N}_{2}$, das allein von der lexikalischen Semantik der beteiligten Nomina gesteuert wird. Hier also bewegen wir uns im Rahmen der vergleichsweise „freien“ Nominalsyntax, die beteiligten lexikalischen Einheiten sind nicht grammatikalisiert. Die Ähnlichkeit mit oder Divergenz von solchen rein lexikalisch-semantisch gesteuerten syntaktischen Konstruktionen und singulativen Komposita werden wir nun in Rechnung stellen. Die Dimensionen dieses Vergleichs sind

- Existenz: Existieren Numerativkonstruktion und singulatives Kompositum oder nur eines von beiden?

- Lexikalisches Spektrum: Bei Existenz beider paralleler Strukturen: Ist das lexikalische Spektrum bei der Kompositabildung ebenso breit und frei wie bei der Numerativkonstruktion?

(i) Dimension der Existenz

Nominalphrasen mit einem singulativen Kompositum als Kern können in der Regel in eine (weitgehend bedeutungsgleiche) Numerativkonstruktion bzw. eine appositive Struktur umgeformt werden:

(7) fünf Schokoladentafeln - fünf Tafeln Schokolade

Dies werten wir als erstes Indiz dafür, dass die Zweitglieder der Komposita hier nicht grammatikalisiert sind. Allerdings sind hier Fallunterschei- 
dungen zu machen; bei den Fallgruppen (i.1) und (i.2) ist der lexikalische Kern ein Konkret-Kontinuativum und bei der korrelierten Numerativkonstruktion handelt es sich um den Typ der Zählkonstruktion, bei (i.3) ist der lexikalische Kern ein Kollektiv-Kontinuativum und es handelt sich um den Typ der Klassifikatorkonstruktion, bei (i.4) ist der lexikalische Kern ein Abstrakt-Kontinuativum.

(i.1) Bei den Singulativa zu Bezeichnungen für dispersive Massen sind (nach Löbel 1986: 26) appositive Strukturen im Singular ungrammatisch, im Plural seien sie ggf. möglich, würden aber beim Zählen eher weniger genutzt:

(8) a. *ein Korn Reis/Staub fünf Körner Reis/*Staub

b. ein Reiskorn fünf Reiskörner/Staubkörner

Ähnlich soll auch gelten:

(9) a. *eine Bohne Kaffee

fünf Bohnen Kaffee

b. eine Kaffeebohne fünf Kaffeebohnen

usw. etwa für Halm Gras/Grashalm, Flocke Schnee/Schneeflocke. Die ausgeschlossenen Ausdrücke sollten aus meiner Sicht jedoch nicht als ungrammatisch verbucht werden. Ihr Ausschluss ist pragmatisch begründet. Das Vorkommen ist keineswegs grundsätzlich ausgeschlossen; so finden sich in den Mannheimer Korpora sowohl ein Halm Stroh als auch eine Bobne Kaffee:

(10) Bevor eine Bohne Kaffee den reichen Verbraucher erreicht, wechselt sie dreißigmal (!) den Besitzer.

(i.2) Bei Singulativa zu Ausdrücken für homogene Massen sind beide Strukturen dagegen im Allgemeinen gleichberechtigt:

(11) a. ein Barren Gold fünf Barren Gold

b. ein Goldbarren fünf Goldbarren

Ähnlich auch: eine Tafel Schokolade - eine Schokoladentafel, ein Laib Brot - ein Brotlaib usw. 
(i.3) Das Verhältnis von Singulativa zu kontinuativen Kollektivsubstantiven (,Genuskollektiven') wie Vieh, Obst, Gepäck und entsprechenden Numerativkonstruktionen scheint von mehreren Faktoren gesteuert zu sein. Man beachte nun, dass in der Extension von Genuskollektiven Individuen durchaus unterschiedlicher Gestalt enthalten sein können, z.B. große und kleine Rinder, sogar Tiere unterschiedlicher Spezies in der Extension eines Vorkommens des Ausdrucks Vieh, Koffer, Taschen, Rucksäcke usw. bei Gepäck. Zählkonstruktionen mit einem Gestaltnomen sind somit nicht geeignet. Es kommen nur Klassifikatorkonstruktionen mit Stück in Frage.

Belebtheit ist einer der steuernden Faktoren:8 Bei Vieh, Wild, Geflügel, also bei Ausdrücken mit belebter Denotation, gibt es die Klassifikatorkonstruktion, aber keine Komposition:

(12) a. ein Stück Vieh

fünf Stück Vieh

b. *ein Viehstück

* fünf Viehstücke

Dieser Aussage scheint die Existenz von Komposita wie Miststück, Dreck(s)stück (mit der Konkurrenzkonstruktion (ein) Stück Dreck) oder Weibstück zu widersprechen: Hier ist in der Regel sogar mit menschlichem Denotat zu rechnen. Allerdings haben diese Singulativa eine stark negativ wertende Komponente als Teil ihrer lexikalischen Bedeutung; es handelt sich um Schimpfwörter. Wie auch hinsichtlich anderer grammatischer Kategorien (man vgl. etwa feminine Genuszuweisung bei Memme, Tunte) verhalten sich Schimpfwörter hier „unkonventionell“ und stellen einen Sonderfall dar.

Die Klassifikatorkonstruktion ist bei belebten Kollektivsubstantiven zahlenmäßig (nach COSMAS-II-Recherchen in den Mannheimer Korpora, die Anfang Juli 2010 durchgeführt wurden) wie folgt belegt:

(13) a. Stück Vieh:

b. Stück Wild:

988 COSMAS-II-Belege

c. Stück Geflügel:

187

Bei nicht-belebtem Erstglied liegen häufig beide Alternativen vor: Stück Obst-Obststück, Stück Schmuck - Schmuckstück, Stück Möbel - Möbelstück.

8 Das oben erwähnte Mann wie in fünf Mann Besatzung/Personal ist selbstverständlich auf menschliche (dazu noch sexusspezifisch männliche) Denotation beschränkt. Auch hier ist Komposition ausgeschlossen. 
Allerdings sind sie meist mit stark unterschiedlicher Frequenz belegt, wie die folgenden Angaben zur Anzahl der Belege zeigen:?

(14)
a. Stück Bekleidung: 5
Bekleidungsstück: ca. 800
b. Stück Besteck: 5
Besteckstück: 2
c. Stück Beute: 12
Beutestück: ca. 1100
d. Stück Gepäck: 16
Gepäckstück: über 3000
e. Stück Geschirr: 7
Geschirrstück: 14
f. Stück Möbel: 13
Möbelstück: ca. 5000
g. Stück Obst: 149
Obststück: ca. 60 (nicht alle einschlägig)
h. Stück Schmuck: 3
Schmuckstück: an die 20.000

Die insgesamt niedrig-frequenten Paare zu Besteck und Geschirr zeigen, dass die Seinsart der beiden Lexeme für Sprecher nicht eindeutig (als kontinuativ) bestimmt ist und daher entsprechende Konstruktionen gemieden werden. Sie können bei der Gesamteinschätzung vernachlässigt werden. Bei Bekleidungsstück, Beutestück, Gepäckstück, Möbelstück und Schmuckstück überwiegen die Komposita stark, die Klassifikatorkonstruktion ist jeweils nur dünn belegt. Interessant hingegen das Paar Obststück versus Stück Obst. Obststück hat zwei Lesarten. Zum einen die im Sinne des Singulativums wie in den folgenden Belegen. Beleg (16) thematisiert die perzeptive Erfassung von Obst und zeigt gleichzeitig den Gebrauch der entsprechenden individuativen und kontinuativen Bezeichnungsformen. Er wird daher ausführlich hier wiedergegeben:

(15) Hunger auf etwas Gesundes? Im Kindertreff Wasbüttel könnt ihr heute euren eigenen Obstsalat zaubern. Jeder bringt einfach zwei Obststücke seiner Wahl mit.

(Braunschweiger Zeitung, 22.05.2006)

(16) Auf dem Gemälde sind Äpfel zu sehen, rote Äpfel. Und ein paar gelbe Birnen. Außerdem grüne Trauben, sowie ein Hand voll blaue Pflaumen. Als der Betrachter aber einen Schritt zurücktritt, das Bild ganz ansieht, erblickt er nicht mehr Äpfel und Birnen, sondern alles zusammen: Er sieht das Stillleben eines Obstkorbs. Zumindest glaubt er das.

Wie Psychologen von der Princeton University jetzt im Wissenschaftsmagazin Science berichten, erliegt der Museumsbesucher aber einer Illusion - er erfasst allein die räumliche Anordnung des Stille-

9 Es wurden, wo nicht anders vermerkt, nur die einschlägigen Belege gezählt. Bei den Komposita gelten die Angaben für das Lemma. 
bens als Ganzes. Die einzelnen Obststücke nimmt er auch jetzt nur nacheinander wahr. Auf einen ersten, flüchtigen Blick kann er also zum Beispiel mehrere rote Äpfel erkennen und auch ihre Lage korrekt angeben. Er erfasst nach einmaligem Hinsehen aber nicht, dass gelbe, blaue und rote Früchte im Korb sind. Und ebenso wenig, dass es dort Obst verschiedener Form gibt: Trauben, Birnen, Pflaumen und Äpfel.

(Die Zeit (Online-Ausgabe), 16.08.2007)

Zum anderen aber kommt häufiger die Lesart ,Teil einer Frucht', ,Stück von einem Obststück' vor wie in:

(17) Langwierig war das Entkernen der Pflaumen oder Zwetschgen, aber noch aufwendiger das Zerkochen und Eindicken der Früchte. Viele Stunden mussten die Helfer rühren, damit die Obststücke im Kessel nicht anbrannten.

(Rhein-Zeitung, 23.09.2008)

Somit kann möglicherweise die vergleichsweise hohe Frequenz der Klassifikatorkonstruktion mit der Vermeidung des ambigen Kompositums erklärt werden. Sollte dies zutreffen, so könnten wir folgende Tendenz festhalten:

Bei Genuskollektiven sind Klassifikatorkonstruktion (mit Stück) und singulatives Kompositum tendenziell komplementär verteilt: Belebte Genuskollektive schlieBen Kompositumbildung aus, nicht-belebte Genuskollektive präferieren die Bildung des singulativen Kompositums.

Bedenken wir nun, (a) dass der Klassifikator Stück (wie in drei Stück Vieh) selbst als grammatikalisiert zu betrachten ist, und (b) dass Klassifikatorkonstruktion und Kompositumbildung tendenziell komplementär verteilt sind, sich also gegenseitig blockieren, so liegt ein grammatikalisierter Status auch für das Kompositionsglied -stück nahe.

(i.4) Bei Abstraktkontinuativa sind Numerativkonstruktionen ausgeschlossen. Weder singularisches Fall noch pluralisches Fälle können als Numerativsubstantiv fungieren. Hier existiert jeweils nur das singulative Kompositum mit dem Zweitglied -fall wie in Todesfall, Unglïcksfall, Glücksfall, Tranerfall:

(18) a. *ein Fall Tod/Glück *fünf Fall/Fälle Tod/Glück b. ein Todesfall/Glücksfall fünf Todesfälle/Glücksfälle 
Auf der anderen Seite ist der Konstruktionstyp

- Fall/Fälle von +Abstraktkontinuativum

zahlreich belegt wie etwa: ein Fall von Etikettenschwindel/Wirtschaftskriminalität/Kindesmissbrauch. Diese Strukturen sind jedoch nicht als Numerativkonstruktionen einzuordnen. Vielmehr geht es hier um die Zuordnung einzelner Erscheinungsformen unter einen Begriff, nicht um die Abbildung nicht-individuierter Situationen auf individuierte, ähnlich auch:

- Instanzen/Realisierungsformen/Exemplare/Inkarnation von Beispiel für, Muster an, ein Ausbund von

Wir nennen dies kurz ,Exemplifikationslesart ${ }^{\star}$ von Fall. Komposita wie Glücksfall, Notfall, Todesfall, Trauerfall und andere lassen eine von-Paraphrase nicht oder nicht bedeutungserhaltend ${ }^{10} \mathrm{zu}$, so dass, selbst wenn wir die von-Konstruktionen als Numerativkonstruktionen werten würden, hier keine Parallele anzusetzen wäre:

(19) *ein Fall von Not/Tod, \$ein Fall von Glück

Andere -fall-Komposita hingegen, etwa Dopingfall, Korruptionsfall, Missbrauchsfall, sind ambig zwischen der Exemplifikationslesart von -fall und der Lesart als Singulativmarker. Singulativität kann, wie gezeigt, bei Abstraktkontinuativa nicht konstruktionell durch eine Numerativkonstruktion kodiert werden. Es bleibt nur Komposition mit -fall. Zusammenfassend können wir bezüglich der Dimension Existenz folgendes Fazit ziehen:

In den Fällen, wo Numerativkonstruktion und singulatives Kompositum (weitgehend) gleichbedeutend nebeneinander existieren, liegt kein Indiz für Grammatikalisierung vor. Dieser Fakt spricht vielmehr für Komposition mit dem $\mathrm{Nu}$ merativsubstantiv.

Wenn singulative Komposita einer bestimmten Fallgruppe nicht mit einer entsprechenden Numerativkonstruktion konkurrieren, sondern sie ersetzen, ist dies ein Indiz für Grammatikalisierung der singulativen Komposita dieser Fallgruppe.

Danach sind die Zweitglieder der singulativen Komposita in den Fallgruppen (i.1) und (i.2) nicht grammatikalisiert, bei (i.3) und (i.4) liegen mit den Zweitgliedern -stück und -fall stärker grammatikalisierte Einheiten vor, die zu reinen Singulativmarkern ausgebleicht sind oder in einer Lesart sein können.

10 Nicht bedeutungserhaltende Umformung wird durch 's' gekennzeichnet. 
(ii) Dimension Lexikalisches Spektrum der Zweitglieder

Der schwache Grammatikalisierungsgrad von Singulativbildungen nach (i.1) und (i.2) wird auch darin deutlich, dass die Köpfe solcher Komposita in Abhängigkeit von dem Modifikator (also dem Kontinuativum) stark variieren. Dies gilt bei allen Komposita mit einem Gestaltnomen als Kopf wie Reiskorn/Hagelkorn/Weizenkorn, Grashalm/Strobhalm (Fallgruppe i.1) oder wie Goldbarren, Brotscheibe/-laib, Holzscheit, Schokoladentafel, Zuckerwürfel usw. (Fallgruppe i.2). Die Zweitglieder drücken nicht (allein) den singulativen Nominalaspekt aus, sondern sie bezeichnen Objekte einer bestimmten Gestalt, und zwar genau jener Gestalt, die auch dem Begriffsinhalt des frei vorkommenden Lexems entspricht. Bei den Singulativbildungen zu Genuskollektiven (Fallgruppe i.3) haben wir - lässt man die fragliche Konstituente -kopf beiseite - nur ein Zweitglied, nämlich -stück. -stück (wie in Beutestück, Gepäcksstück, Möbelstück) hat keinen weitergehenden begrifflichen Inhalt, es erschöpft sich in der grammatischen Bedeutung der Markierung des singulativen Aspekts. ${ }^{11}$

Auch bei Singulativbildungen zu Abstrakta (Fallgruppe i.4) haben wir nur ein Zweitglied zur Verfügung, nämlich -fall, das wir ebenfalls als reinen Singulativmarker betrachten können. Wir halten daher fest:

-fall und -stück sind die am stärksten grammatikalisierten Singulativmarker. -fall fungiert in dieser Verwendung als Singulativmarker für die Herleitung individuativer, Abstraktsubstantive (Ereignisbezeichnungen) aus kontinuativen Abstraktsubstantiven, -stück leitet aus kontinuativen Kollektivsubstantiven (Genuskollektiva), die in der Regel nicht-belebt sind, individuative Bezeichnungen für Elemente entsprechender Kollektionen ab.

\section{2 -fall als Singulativmarker}

Im Folgenden sollen noch kurz einige Besonderheiten dieser Verwendung von -fall betrachtet werden. Für den stärkeren Grammatikalisierungsgrad gibt es weitere Indizien, die -fall mit den stärker grammatikalisierten Kollektivmarkern wie -gut, -werk, -zeug teilt. Wie diese akzeptiert -fall auch nicht substantivische Erstglieder wie in Störfall (verbales Erstglied, vgl. Streugut), Ernstfall (adjektivisches Erstglied, vgl. Grünzeug), Zwischenfall (präpositionales Erstglied, vgl. Beiwerk) und es kann sogar redundant bzw. leer

11 Auch in der komplementär verteilten Numerativkonstruktion (drei Stück Vieb) kommt nur diese grammatische Funktion zum Tragen. Die Lesarten des Lexems wie es außerhalb dieser Konstruktion vorkommt, können begrifflich reicher sein: ,Teil eines konkreten Objekts' (vgl. Er hat ein Stück von der Wurst abgeschnitten, auch in Komposita wie Hautstück, Käsestück), ,Theaterstück' (vgl. Es wurden drei neue Stücke aufgeführt); vgl. auch Abschnitt 5. 
angewendet werden. D.h. es ist auch auf individuative Ereignisbezeichnungen anwendbar wie in Mordfall, Diebstablsfall, Brandfall, Einsatofall.

Einzuräumen ist allerdings, dass die Grammatikalisierung zum Singulativmarker für Ereignissubstantive nicht vollständig vollzogen ist. Dafür können verschiedene Gesichtspunkte geltend gemacht werden:

a) Bei vielen Bildungen gibt es - auch wenn wir von der nur unscharfen Abgrenzung gegenüber der Exemplifikationslesart absehen - einen semantischen Mehrwert: nicht jedes Glücks- bzw. Unglücksereignis ist ein Glücksfall bzw. Unglücksfall. Häufig gibt es - in der Nachfolge von lat. casus - auch noch eine Art juristische Konnotation wie etwa bei Mordfall, Schaden(s)fall.

b) Viele Bildungen werden gar nicht oder kaum in Verbindung mit Numeralia gebraucht, erfüllen somit nicht den Zweck der Zählbarmachung, sondern in Konditionalsyntagmen mithilfe von im wie bei im Gefahrenfall, im Bejabungsfall (vgl. Ortner et al. 1991: 178, „Papierdeutsch"), im Krankheitsfall. Hier ist der erzeugte Denotatstyp auch nicht unbedingt ein Ereignis im engeren Sinne, sondern eine Situation, eine Eventualität.

c) Viele Bildungen werden nicht oder nicht nur mit der Denotation von Einzelereignissen gebraucht, sondern als Personenbezeichnungen (Pflegefall, Härtefall, Sozialfall), vor allem im administrativen Kontext (vgl. auch Zifonun 2009).

All diese Störeffekte sozusagen mögen mit dazu beigetragen haben, dass die grammatische Funktion von -fall als Singulativmarker für Ereignissubstantive bisher nicht beachtet wurde. Ortner a.a.O. ordnen -fall-Komposita dem Typ der „verdeutlichenden Gleichsetzungskomposita“ zu, mit den Bestandteilen definierte - definierende Größe, also etwa: Schadensfall ,Fall, der darin besteht, dass ein Schaden auftritt'. Dabei wird eine gewisse grammatische Funktion durchaus gesehen, sie sprechen (vgl. S. 176f) davon, solche Bildungen hätten die Funktion, den „Bestand an grammatischen Abstraktbildungen" zu ergänzen und ggf. Abstraktableitungen etwa mit - ung oder -ion zu monosemieren. Nun ist aber dieser undeutliche Charakter typisch für Grammatikalisierungsphänomene insgesamt und insbesondere auch in der Wortbildung gang und gäbe. So ist bei den Elementen -gut und -zeug auch eine Komponente erkennbar, die über die reine Kollektivmarkierung hinausgeht. Wellmann (1969: 86ff) spricht hier jeweils (für -gut, bei Streugut, Mablgut) von „eine(r) Vielheit als Material für einen 
bestimmten Zweck" bei -zeug spricht Wellmann (1975: 441) (in Schreibzeug, Nähzeug, Putzzeug) von einem Mittel zur Bildung von Instrumentativa. Beide Elemente werden daneben aber auch mit substantivischen und adjektivischen Basen gebraucht, wo die instrumentale Note nicht zur Anwendung kommt oder kommen kann, dafür aber andere konnotative Restriktionen gelten.

\section{Kollektiva}

Kollektivbildungen mit den Markern -gut, -werk und -zeug sind vergleichsweise stärker grammatikalisiert. Indizien dafür sind:

1. Selbstständiges Gut/Werk ist individuativ (pluralisierbar), als Kollektivmarker dagegen kontinuativ (nicht pluralisierbar), selbstständiges Zeug ist bereits kontinuativ. ${ }^{12}$

2. Die Marker können nicht selbstständig als Kopf einer appositiven NP bzw. Numerativkonstruktion verwendet werden: *ein Werk/Zeug Zucker, *ein Gut Lied(er). Numerativkonstruktion und komplexes Wort existieren also nicht nebeneinander. Anders ist dies z.B. bei den Kollektiva -herde, -büschel, -stapel als Zweitglied:

(20) a. eine Herde Schafe

fünf Herden Schafe

b. eine Schafherde fünf Schafherden

(21) a. ein Büschel Gras

fünf Büschel Gras

b. ein Grasbüschel

fünf Grasbüschel

(22) a. ein Stapel Bücher

fünf Stapel Bücher

b. ein Bücherstapel

fünf Bücherstapel

Diese Einheiten sind sowohl als Simplizia in der Numerativkonstruktion, als auch als Zweitglieder pluralisierbar und mit Zahlwörtern verbindbar und somit individuativ: Die Individuen, die sie bezeichnen, sind Kollektionen, die ihrerseits aus elementaren Einzelindividuen (wie in Herde Schafe/Stapel Bücher) oder auch aus dispersiven

12 Interessanterweise gibt es Zusammensetzungen mit -zeng, die sowohl als Kontinuativum wie als Individuativum einzuordnen sind wie Spielzeug, Werkzeug. Man kann hier die kontinuative Kategorie (entsprechend dem Simplex) als zugrundeliegend betrachten, die individuative ist eine Singulativbildung ohne morphologischen Ausdruck, also ohne Singulativmarker. 
Massen (wie in Büschel Gras) bestehen. Diese Elemente verhalten sich also ganz parallel zu -korn, -halm, -bohne, -barren usw. bei den Singulativa. Sie sind nicht grammatikalisiert.

Man mag nun argumentieren, bei kontinuativen Markern wie -gut, werk, -zeug sei die auf Individuation ausgerichtete Numerativkonstruktion ohnehin nicht erwartbar. Dem kann aber entgegengehalten werden, dass vergleichbare appositive Strukturen auch existieren, wenn keine Zählbarkeit gegeben ist, sondern nur auf die Größe einer Kollektion abgehoben wird. Dies gilt z.B. für die Elemente Haufen, Menge, Masse oder auch Berg in:

(23) ein (großer) Haufen/Berg Probleme - Haufen/Berge von Problemen - *fünf Haufen/Berge von Problemen

(24) eine (große) Masse/Menge Probleme - (große) Massen/Mengen von Problemen - *fünf Massen/Mengen von Problemen

Vorstellbar wäre es somit, dass ähnlich wie ein Haufen Unsinn auch *ein Gut Unsinn $n^{13}$ als nicht-zählbare appositive Kollektivkonstruktion gebraucht würde.

3. Im Gegensatz zu den individuativen Markern -berde, -büschel, -stapel usw. liegt bei -gut, -werk, -zeug keine lexikalische Differenziertheit des Zweitglieds in Abhängigkeit vom Erstglied vor. Während bei ersteren wieder die Gestalt der Kollektion für Distinktheit sorgt, geben letztere keine Auskunft über solche Gestalteigenschaften. Daher gibt es zu manchen Erstgliedern auch Doubletten mit -werk oder -zeug: Schubwerk - Schubzeng, Zuckerwerk - Zuckerzeug.

4. Wie der Singulativmarker -fall werden neben substantivischen Erstgliedern auch Verb-, Adjektiv- und Präpositionalstämme in das Muster einbezogen: Grillgut/Trockengut, Naschwerk/Beiwerk, Scbreibzeng/Grünzeng/Unterzeng.

5. Marginal besteht auch die Möglichkeit der leeren/redundanten Anwendung: So liegt mit Gesimswerk (Wellmann 1969: 79) eine Kollektivbildung zu dem Kollektivum Gesims vor.

13 Man denke z.B. an engl. lot, wörtlich, Los, Posten' in seiner numerativen Funktion wie z.B. in a lot of fun. 


\subsection{Zur Problematik von ,Kollektion“ allgemein:}

die Kombinatorik semantischer Klassenmerkmale

Begriffsbildung über Kollektion ist ein vergleichsweise komplexes Verfahren, bei dem mehrere „Bedeutungskomponenten“ (Kuhn 1982: 91) oder -aspekte zum Tragen kommen können. Kuhn unterscheidet für das Deutsche die drei Komponenten ,Elementqualität', ,Vereinigungsqualität ${ }^{6}$ und ,Gegenstandscharakter': Alle drei Komponenten sind in einem prototypischen Kollektivum wie Gebirge oder Gebüsch positiv spezifiziert. Bei Gebirge/Gebüsch ist gefordert, dass jedes Element der Kollektion eine bestimmte Qualität hat, nämlich dass es sich um einen Berg bzw. einen Busch handelt. Eine solche Elementqualität ist z.B. bei Gruppe, Horde oder auch Sippe, Nation nicht gefordert. Bei Gebirge/Gebüsch ist außerdem eine bestimmte Qualität gefordert, die die Vereinigung der Elemente zu einer Kollektion bewirkt, hier die Bedingung einer räumlichen Nähe oder Verbundenheit. Man kann hier auch von ,Übersummativität' sprechen, in dem Sinne, dass die Kollektion mehr ist als die Summe der einzelnen Teile. Eine entsprechende Vereinigungsqualität ist z.B. bei Adel, Arbeiterschaft (oder anderen -schaft-Kollektiva) nicht gefordert. Hier ist die Elementqualität ,adelig sein/Arbeiter sein' ausreichend, um die Zugehörigkeit zur Kollektion zu etablieren. Schließlich ist bei Gebirge/Gebiusch aufgrund der Vereinigungsqualität ein Abgrenzungs- bzw. Identitätskriterium gegenüber anderen Kollektionen mit gleicher Elementqualität gegeben: Es gibt verschiedene Gebirge, zu denen jeweils verschiedene Elemente (Berge) gehören. Die gebört-zu-Paraphrase (Dieser Berg da vorn gehört zum Fichtelgebirge) zählt als Indiz für den Gegenstandscharakter. Pluralisierbarkeit im Sinne eines Mengenplurals (die verschiedenen Gebirge) setzt somit Gegenstandscharakter voraus, aber nicht immer ist Gegenstandscharakter mit Pluralisierbarkeit verbunden. Man denke etwa an Arbeiterschaft, Bürgertum. gebörtzu-Aussagen sind hier möglich (Karl gehört zur Arbeiterschaft/ zum Bürgertum), Pluralisierung dagegen ist ausgeschlossen. Dies ist auf die mangelnde Vereinigungsqualität zurückzuführen. ${ }^{14}$

Je nach Belegung dieser drei Komponenten sind (nach Kuhn) 12 Klassen von Kollektivsubstantiven zu unterscheiden.

Die beiden Pole bilden eindeutig individuative Kollektivsubstantive (zählbar, pluralfähig usw.) wie Gruppe, Mannschaft auf der einen Seite und eindeutig kontinuative Kollektivsubstantive wie Vieh, Laub, Obst. Bei den

14 Das bedeutet nicht, dass alle -schaft- oder -tum-Ableitungen nicht pluralfähig sind. Pluralfähiges Mannschaft ist geradezu ein Paradebeispiel für Vorliegen von Vereinigungsqualität. 
prototypischen Vertretern der individuativen Kollektiva liegt folgende Merkmalskombination vor:

- (pluralfähige) individuative Kollektivsubstantive: [+Vereinigungsqualität], [+ Gegenstandscharakter]

Das Vorliegen von Vereinigungsqualität bzw. Übersummativität ist ablesbar daran, dass die einzelnen Elemente der Kollektion nicht unter den entsprechenden Begriff fallen, also keine distributive Referenz (vgl. Bunt 1985: 20) vorliegt:

- wenn [+Vereinigungsqualität ] dann [-distributive Referenz]

Es gilt z.B. nicht: Jeder Teil einer Gruppe, Mannschaft oder Nation ist eine Gruppe, Mannschaft oder Nation.

Bei den prototypischen Vertretern der kontinuativen Kollektiva liegt die komplementäre Merkmalskombination vor:

- kontinuative Kollektivsubstantive:

[-Vereinigungsqualität], [- Gegenstandscharakter]

Mangelnde Vereinigungsqualität äußert sich in distributiver Referenz:

- wenn [-Vereinigungsqualität ] dann [+distributive Referenz]

Es gilt: Jeder Teil von Vieh, Laub oder Obst (einer Ansammlung von Vieh/Laub/Obst) ist Vieh bzw. Laub oder Obst.

Allerdings sind andere Kollektivkontinuativa durchaus mit Vereinigungsqualität ausgestattet: Bei einer einzelnen Feder wird man nicht von Gefieder sprechen, bei einem einzelnen Balken nicht von Gebälk, bei einem einzelnen Ast nicht von Geäst; bei einer Bluse kaum von Bekleidung. Daraus kann man schließen, dass mangelnde Vereinigungsqualität kein notwendiges Merkmal von Kollektivkontinuativa ist, während wir fehlenden Gegenstandscharakter als notwendig einzuschätzen haben. 
4.2 Die Kombinatorik semantischer Klassenmerkmale bei - gut, -werk und -zeug

Daran schließt sich nun die Frage an, wie es mit den hier im Zentrum stehenden Kollektivmarkern steht. Zeigen sie bezüglich dieser Merkmalsverteilungen ein jeweils eindeutiges oder zumindest systematisches Verhalten? Wir vertreten folgende These:

Die drei aus Kompositionsbestandteilen grammatikalisierten Kollektivmarker des

Deutschen -gut, -werk, -zeug erzeugen grundsätzlich Kollektivssubstantive ohne

Gegenstandscharakter, somit Kontinuativa. In Abhängigkeit vom Typ des Erstglieds kann jedoch Vereinigungsqualität vorliegen.

Bei allen drei Markern ist die Kategorie des Erstgliedes ausschlaggebend für das Vorliegen oder Nicht-Vorliegen von Vereinigungsqualität. Bei einem individuativen substantivischen Erstglied hat das Wortbildungsprodukt bei allen drei Markern Vereinigungsqualität wie etwa bei Liedgut/Sagengut (eines Volkes), Gedankengut/Ideengut (einer Person), Maververk (eines Gebäudes), Bettzeng, Verbandszeug. Bei -zeng ist auch bei verbalem Erstglied mit Vereinigungsqualität zu rechnen: Flickzeug, Malz̨eug, Nähzeug, Turnzeng, Schreibreug, Waschreug bezeichnen jeweils Kollektionen meist unterschiedlicher Gegenstände, die zusammen den mit dem Verb ausgedrückten Zwecken dienen. Verbales Erstglied bei -gut und -werk führt hingegen nicht zum Vorliegen von Vereinigungsqualität: Grillgut, Streugut, Naschwerk, Treibgut bezeichnen jede auch noch so kleine Ansammlung von zu Grillendem, Auszustreuendem, zu Naschendem oder (auf einem Gewässer) Treibendem. Ein Minimalpaar stellen Waschgut (,alles zu Waschende) und Waschzeug (,was man zum Waschen braucht') dar. Kontinuative substantivische Erstglieder sind im Allgemeinen mit Fehlen von Vereinigungsqualität korreliert wie etwa bei Steingut, Tongut; Zuckerwerk; Dreckszeng, Giftzeng, Plastikzeng, Steinzeng. ${ }^{15}$ Erstglieder anderer Wortarten, vor allem Adjektive, korrelieren in der Regel mit fehlender Vereinigungsqualität: Leergut, Trockengut; Grobzeug, Grünzeug.

Auch diese Tatsache einer vergleichsweise systematischen Verteilung der subdifferenzierenden semantischen Klassenmerkmale spricht für die Grammatikalisierung der Elemente.

15 Bei - zeug gibt es eine spezifische Reihenbildung im Sinne von ,Bekleidung (bei bestimmten Gelegenheiten, aus bestimmten Materialien)'. In dieser Lesart liegt auch bei kontinuativem Erstglied Vereinigungsqualität vor wie etwa bei Regenzeug, Lederzeug, entsprechend wird auch Unterzeug interpretiert. 


\section{Halbaffix oder Kompositionsglied?}

Sollten die hier diskutierten Singulativ- und Kollektivmarker als Halbaffixe/Affixoide, speziell als Halbsuffixe/Suffixoide eingeordnet werden oder handelt es sich um besondere Verwendungen der entsprechenden Lexeme als Kompositionsglieder, speziell als Köpfe von Komposita? (Auf Halbpräfixe/Präfixoide wird im Folgenden nur am Rande eingegangen.)

In Darstellungen der deutschen Wortbildung gehen die Einordnungen deutlich auseinander. So wird in Fleischer \& Barz (1995) und Motsch (2004), die Affixoide grundsätzlich ablehnen, mit Bezug auf -werk von einem Suffix gesprochen, bei -gut und - reug dagegen vom Zweitglied eines Kompositums. Fleischer \& Barz (1995: 143) weisen immerhin darauf hin, dass in Fleischer (1983) diese beiden Elemente noch als Suffixe eingeordnet wurden.

Wellmann (1975) ordnet dagegen -werk als Suffixoid ein (S. 419), bei -gut spricht er von „Funktionalisierung des Kompositionsglieds“ (S. 167), -zeug nennt er ,suffixartig“ (S. 166).

Ortner et al. (1991: 252) haben einen sehr weiten Suffixoid-Begriff. Dort gilt nicht nur -gut (in Ideengut), sondern auch -volk (in Bienenvolk), -welt (in Frauenwelt), -reich (in Tierreich), -komplex (in Fragenkomplex) usw. als Suffixoid.

Für Halbaffixe ${ }^{16}$ wird zunächst üblicherweise ein lexikalisches Kriterium geltend gemacht. Danach wird dann von Halbaffixen gesprochen, wenn a) neben der fraglichen Einheit homophone Lexeme mit anderer, aber nachvollziehbar verwandter Bedeutung existieren wie die Adjektive arm, frei, los, müde, voll zu schadstoffarm, wartungsfrei, haltlos, reformmüde oder geschmackvoll, oder wenn b) sofern es keine freien Vorkommen gibt, zumindest die fraglichen Einheiten strukturell (z.B. als Suffixableitungen zu einem Substantiv- oder Verbstamm) einem Lexem entsprechen wie -artig, -formig, -haltig in explosionsartig, flaschenformig, salzhaltig. Die Einheiten, mit denen wir uns hier beschäftigten, gehören zu Typ a). Dieses Kriterium verknüpft die Halbaffixe mit Kompositionsgliedern und grenzt es von Affixen ab. Wir beziehen uns im Folgenden schematisch so auf dieses Kriterium:

Lexikalisches Kriterium:

wenn Existenz eines homophonen Lexems (Fälle a) und b)), dann [-Suffixstatus] wenn keine Existenz eines homophonen Lexems, dann [+Suffixstatus]

16 Im Folgenden wird aus praktischen Gründen nur noch der Terminus ,Halbaffix‘ bzw. ,Halbsuffix' gebraucht. 
Halbaffixe; die dieses lexikalische Kriterium erfüllen, unterscheiden sich auch in ihrer morphophonologischen Struktur nicht oder zumindest nicht grundsätzlich von Kompositionsgliedern:

1. Halbaffixe konstituieren wie Kompositionsglieder ein eigenes phonologisches bzw. prosodisches Wort: Trauerfall unterscheidet sich prosodisch nicht von $W$ asserfall.

2. Halbaffixe können bis zu zweisilbig sein und dabei die kanonische Silbenstruktur jeweils von Einsilblern (mit vollvokalischem Nukleus) oder Zweisilblern (mit Haupt- und Schwa- oder i-haltiger Nebensilbe) aufweisen.

3. Halbsuffixe lizensieren wie Kompositionszweitglieder erweiterte Stammvarianten des Erstglieds: Ideengut wie Ideenklau, Teufelszeug wie Tenfelskë̈che, Ladegut wie Ladefläche (zum erweiterten Verbstamm lade-).

Diese morphophonologischen Kriterien, so sollte man annehmen, grenzen die Halbaffixe scharf ab von den „echten“ Affixen/Suffixen. Wir formulieren kurz so:

Morphophonologisches Kriterium:

wenn (nach 1. bis 3.) Struktur wie beim Kompositum, dann [-Suffixstatus]

wenn (nach 1. bis 3.) Struktur abweichend vom Kompositum, dann [+Suffixstatus]

Nach diesen Kriterien (vgl. dazu Raffelsiefen demn.) ${ }^{17}$ zählen aber auch Einheiten wie -bar, -sam, -schaft, -tum zu den Halbaffixen: Sie haben die Vokale /a/, und /u/ als Silbennukleus (sowie /o/ wie in -los), nicht wie die echten konsonanteninitialen Suffixe /a/ oder /i/ (vgl. Punkt 2.). Sie selegieren teilweise erweiterte Stammvarianten wie in Bauernschaft, Volkstum; -bar und -sam lizensieren zwar nur nicht-erweiterte Stämme (vgl. tragbar - *tragebar, ratsam - *ratesam), aber keine umgelauteten Varianten (vgl. *trägbar, * rätsam - erträglich, rätlich).

Suffixe hingegen bilden keine eigenen phonologischen Wörter: Vokalinitiale Suffixe (wie -er, -ig, -ung) sind integriert in das phonologische Wort, solche mit konsonantischem Anfangsrand konstituieren eigene Silben außerhalb des phonologischen Wortes, was mit spezifischen „Grenzeffek-

17 Raffelsiefen (demn.) geht von folgender Liste der Halbsuffixe aus: -bar, -sal, -baft, -schaft, -fach, -falls, -sam, -beit, -keit, -lei, -weise, maßen, -bold, -los, -tum, -mut. Definitorisch sind bei ihr allein morphophonologische Kriterien, die die ihrerseits mit semantischem bleaching korreliert sind. 
ten" verbunden ist. Letztere sind einsilbig und weisen in der Regel einen reduzierten Schwa-/-i-Vokalismus auf (vgl. etwa das Adjektivsuffixe -lich und die Substantivsuffixe -chen, -nis, -[lein], -ling). Sie lizensieren nichterweiterte Stammvarianten der Basis (vgl. glïcklich, Teufelchen), ggf. auch Varianten des Stammes mit Umlaut (Häuschen, rötlich, Täufling). Die folgende Tabelle zeigt die Unterschiede im Vokalismus (Punkt 2.) und in der Wahl der Stammvariante (Punkt 3.) anhand der Elemente (in alphabetischer Reihenfolge): -bar, -chen, -fall, -gut, -ler, -lich, -ling, -los, -nis, -sam, -schaft, -tum, -reug:

\begin{tabular}{|c|c|c|c|c|}
\hline \multirow{2}{*}{ Elemente } & \multirow{2}{*}{ Vokalismus } & \multicolumn{3}{|c|}{ Stammvariante } \\
\hline & & tumgetautet & unerweitert & erweitert \\
\hline $\begin{array}{l}\text {-chen, -ler, } \\
\text {-lich, -ling, } \\
\text {-nis }\end{array}$ & $\begin{array}{l}\mid \mathrm{a} / \\
/ \mathrm{i} /\end{array}$ & $\begin{array}{l}\text { Hänschen, } \\
\text { Händles, rötlich, } \\
\text { Täugling, } \\
\text { Besänfnis }\end{array}$ & & \\
\hline -bar, -sam & \multirow{4}{*}{$\begin{array}{l}\text { /a/ } \\
/ \mathrm{o} / \\
/ \mathrm{u} /\end{array}$} & & $\begin{array}{l}\text { tragbas; } \\
\text { strebsam }\end{array}$ & \\
\hline -fall, -schaft & & & & $\begin{array}{l}\text { Glücksfall, } \\
\text { Bauernschaft }\end{array}$ \\
\hline -los, -zeug & & $=$ & 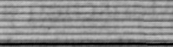 & arbeitslos, Teufelszeug \\
\hline$-g u t,-t u m$ & & & & $\begin{array}{l}\text { Diebesgut, Ideengut, } \\
\text { Volkstum }\end{array}$ \\
\hline
\end{tabular}

Schraffierung: Domäne der Derivation

graue Schattierung: Domäne der Komposition

Nun werden aber traditionell Einheiten wie -bar, -sam, -schaft, -tum bedenkenlos den Suffixen zugerechnet. Dies ist dem Primat des lexikalischen Kriteriums über die morphophonologischen Kriterien für den Halbaffix-Status zuzuschreiben: Da diese Einheiten synchron keine Verbindung mehr haben zu einem Lexem, scheint die Einordnung als Halbaffix nicht in Frage zu kommen.

Flankierend zu dem lexikalischen Kriterium, also der reinen Existenz eines homophonen Lexems, wird üblicherweise ein semantisches Kriterium geltend gemacht, das die Halbaffixe mit den Affixen verbinde, nämlich die gegenüber Lexemen „wenig spezifische Bedeutung“. Dem kann jedoch (vgl. z.B. Kastovsky 2009: 327) entgegengehalten werden, dass Affixe neben grammatischer Bedeutung (wie bei den Nomina agentis bzw. patientis bildenden Affixen -er und -ling) sehr wohl auch spezifische 
Bedeutungen haben können (wie etwa mono- ,eins' oder $b i{ }^{-}$, ,zwei'). Nun ist aber andererseits nicht zu bestreiten, dass wir bei einem direkten Vergleich z.B. zwischen freien und gebundenen Verwendungen von los deutliche Ausbleichung bei Bindung, also weniger spezifische Bedeutung, erkennen können. los kommt nur prädikativ (als Adkopula im Sinne der IDSGrammatik) und mit obligatorischer Komplementation durch einen Akkusativ vor wie in:

(25) Ich bin ihn endlich los. Er war die Arbeit los.

Mit freiem los ist immer die Bedeutung des (ggf. billigend in Kauf genommenen) Verlustes, eine negative Wertung verbunden. Dies fehlt in der gebunden Verwendung völlig. Hier heißt -los nur, ohne'.

(26) arbeitslos, akzentlos, alternativlos, furchtlos, hemmungslos, schutzlos

Bei den hier interessierenden Singulativ- und Kollektivmarkern liegen mit -gut, -stück, -werk und -reug Ausdrücke vor, die als Lexeme eine sehr allgemeine Grundbedeutung haben. Gut geht auf die Konversion des Adjektivstammes zurück, um, wie das DWB formuliert „die verschiedenen Bedeutungen des Adjektivs ins Gegenständliche zu erheben“. Es bedeutet also zunächst, was dem Menschen angenehm, erfreulich, vorteilhaft ist'. Diese Lesart findet sich vor allem in der prädikativen Verwendung

(27) $\mathrm{X}$ ist ein hohes/wichtiges/das höchste/Gut (,summum bonum)

sowie in Zusammensetzungen wie Kulturgut. Daneben gibt es semantisch angereicherte Verwendungen wie die heute frequente Lesart, unbeweglicher Besitz, Grundbesitz', die auch in Zusammensetzungen wie

(28) Bauerngut, Hofgut, Kirchengut, Landgut, Rittergut, Weingut

vorliegt, daneben die - im Simplex nur bei pluralischem Güter, in Zusammensetzungen wie Eilgut, Frachtgut, Gefahrgut, Luxusgut, Transportgut auch im Singular vorliegende - Lesart, Ware'.

Bei Werk ist zunächst von der allgemeinen Bedeutung, Tätigkeit, Arbeitsleistung' (vgl. z.B. Gemeinschaftswerk, Versöbnungswerk) auszugehen mit Anreicherungen und Spezialisierungen wie ,Schöpfung der Kunst usw.‘ wie in den Komposita Jabrhundertwerk, Kunstwerk, Lehrwerk oder ,bauliche Anlage/Betriebsanlage' wie in Gaswerk, Kraftwerk, Opelwerk, Pumpwerk, Stablwerk. 
Der allgemeinen Bedeutung von Stück, die zurückgehend auf die Wurzel steug ,stoßen, schlagen, abhauen' als ,(abgetrennter) Teil eines konkreten Objekts' (wie in Endstück) beschrieben werden kann, stehen in vielfältiger Weise angereicherte Lesarten zur Seite, vor allem die im Kompositum frequente ,literarisches Erzeugnis, auf der Bühne dargestellte Dichtung, Theaterstück' wie in Ballettstück, Einpersonenstück, Skandalstück.

Stellen wir also in Rechnung, dass die Simplizia hier oft neben der allgemeinen Grundbedeutung semantisch angereicherte Lesarten ausgebildet haben, die auch in Komposita sehr produktiv waren. Wir sollten dann, wenn es darum geht, die weniger spezifische Bedeutung für das Halbaffix nachzuweisen, nicht von diesen lexikalischen Anreicherungen ausgehen, sondern von den Grundbedeutungen. Aber auch diesen gegenüber, so behaupte ich, liegt hier eine weitere Ausbleichung vor, eine Reduktion auf die nominal-aspektuale Bedeutung. Diese Reduktion auf die grammatische Bedeutung ,Erzeugung eines bestimmten Nominalaspekts' stellt innerhalb des breiten Spektrums dessen, was als ,jeweils weniger spezifische Bedeutung' betrachtet wird, den klar umrissenen Pol der minimal spezifischen Bedeutung dar. Dies ändert nichts an der Tatsache, dass anders als der Terminus ,Halbaffix' suggeriert, das semantische Kriterium grundsätzlich nicht dazu geeignet ist, den semantischen Abstand zwischen Affixbedeutung und Bedeutung des Lexems als Teil eines Kompositums in zwei disjunkte Abschnitte aufzuteilen, an deren Grenze (idealiter in der Mitte dieses Abstandes) die Halbaffixbedeutung anzusiedeln wäre. Eben dieses Fehlen einer scharfen Grenze oder auch die von Einzelfall zu Einzelfall unterschiedliche semantische Relation zwischen Bedeutung des Lexems (als Teil eines Kompositums) und präsumptiv weniger spezifischer Bedeutung des potentiellen Halbaffixes ist ein Hauptargument gegen die Etablierung der Kategorie Halbaffix selbst. So heißt es bei Booij (2009: 209):

The analytical problem that there is no sharp boundary between compounding and affixal derivation is not solved by postulating a category of semi-affixes or affixoids. That is just a convenient description of the fact that the boundary between compounding and derivation is blurred, but does not in itself provide an explanation of why this is the case.

Um die Verwirrung bezüglich der semantischen Eigenschaften von Halbaffixen vollständig zu machen, argumentiert Booij an dieser Stelle anscheinend genau entgegengesetzt zu Kastovsky. Aufgrund von Reihenbildungen wie ndl. groente-boer ,Gemüsehändler' (wörtl. ,Gemüse-Bauer'), sigaren-boer ,Zigarenhändler' usw. zum Simplex boer ,Bauer' oder steen-koud, steen-goed, steen-rijk ,sehr kalt/gut/reich' (wörtl. ,stein-kalt' usw.) zum Simplex steen ,Stein`spricht Booij bei den präsumptiven Affixoiden oder Halbaffixen von 
[...] morphemes which look like parts of compounds, and do occur as lexemes, but have a specific and more restricted meaning when used as parts of compound. (Booij 2009: 208, Hervorhebung G.Z.) ${ }^{18}$

Während bei Kastovsky wenig spezifische Bedeutung als Merkmal von Affixen genannt wird, das, so könnte man folgern, auch von Halbaffixen gelten müsste, spricht Booij von spezifischer Bedeutung als HalbaffixMerkmal. Der Widerspruch mildert sich jedoch, wenn die jeweils unterschiedlichen Lesarten von ,spezifisch' hier in Rechnung gestellt werden. Während Kastovsky mit spezifisch meint ,reich an begrifflichen Merkmalen', verwendet Booij spezifisch rein relational für das Verhältnis zwischen Lexem und Halbaffix: sperifisch heißt dann , abweichend von der Lexembedeutung،. Zusammen mit der Bestimmung der Halbaffix-Bedeutung als gegenüber der Lexembedeutung restringierter, lässt sich dann doch ein vages semantisches Verständnis von Halbaffixen gewinnen, bei dem die beiden Auffassungen miteinander kompatibel erscheinen.

Wenn auf der semantischen Ebene die Kategorie der Halbaffixe somit fragwürdig erscheint, so könnte man sich, um die Kategorie zu retten, auf die morphophonologische Ebene zurückziehen, die vergleichsweise klare Abgrenzungen liefern könnte. Aber auch hier gibt es verschiedene Möglichkeiten: Wenn (um uns auf Bildungsmittel für Substantive zu beschränken) sowohl Elemente wie -schaft und -tum als auch -gut und -fall, die jeweils nach diesem Kriterium [-Suffixstatus] aufweisen, als Halbaffixe eingestuft werden sollen, dann muss zusätzlich das lexikalische Kriterium und eine Version des semantischen Kriteriums herangezogen werden, da sonst Elemente wie -gut und - fall gar nicht als Halbaffix-Kandidaten in den Blick geraten würden, sondern schlicht Kompositumglieder wären.

Beschränkt man sich andererseits auf Elemente ohne homonyme Lexeme wie -schaft, -tum, so läge es näher, verschiedene Suffixtypen mit unterschiedlichen phonologisch-prosodischen Eigenschaften zu unterscheiden, statt eine intermediäre Kategorie des Halbaffixes einzuführen. Diese setzt ja in gewisser Weise die Idee der Grammatikalisierung von Kompositumsgliedern zu Affixen voraus, auch wenn sie dem nichtdiskreten, sondern graduellen oder vielfach abgestuften Charakter des Prozesses nicht gerecht wird. Redet man also von Halbaffixen, so können die Elemente, die dem Ausgangszustand Kompositumglied noch nahe sind, nicht einfach ausgeschlossen werden.

Ich ziehe aus dieser Argumentation folgenden Schluss: Die Existenz von Elementen, die sowohl Eigenschaften von Affixen als auch von Komposi-

18 Ähnlich ist auch die Konzeption in Van Goethem (2008) mit Bezug auf ndl. und frz. Adjektive, die zu Präfixen grammatikalisiert werden. 
tionsgliedern haben, ist nicht zu bestreiten. Wünschenswert ist eine möglichst differenzierte, z.B. nach den hier genannten Kriterien (lexikalisches Kriterium, morphophonologisches Kriterium, semantisches Kriterium) gegliederte Erfassung und Ordnung der unterschiedlichen Erscheinungsformen. Gegen die Benennung Halbaffix als Sammelbezeichnung für alle Übergangsphänomene (oder auch je nach Interessenlage nur für bestimmte) ist nichts einzuwenden. Es sollte aber bei Bezug auf das ganze Spektrum klar sein, dass damit nicht eine Kategorie benannt ist, für die eine bestimmte Menge von Merkmalen gilt, d.h. es ist dann nicht der Fall, dass alle Elemente, die als Halbaffixe bezeichnet werden, über eine gemeinsame Schnittmenge an Merkmalen verfügen würden.

Dieser Sachverhalt wird deutlich, wenn wir Halbaffix-Kandidaten die Singulativ- und Kollektivmarker, die hier im Zentrum stehen und weitere Elemente - nach den vorgeschlagenen Kriterien bewerten und ordnen. Dazu schlagen wir auch für das semantische Kriterium - trotz der oben diskutierten Risiken dieses Vorgehens - eine Bewertung nach dem semantischen Suffixstatus vor, bei der nur die beiden Pole Berücksichtigung finden:

\section{Semantisches Kriterium:}

wenn grammatische Bedeutung, dann [+Suffix]

wenn keine grammatische Bedeutung, dann [-Suffix]

Dieser reduktionistische Umgang mit der Semantik ist mit dem hier behandelten Phänomen der Singulativ- und Kollektivmarkierung zu rechtfertigen, bei dem es um grammatische Bedeutung geht.

Die folgende Tabelle zeigt die möglichen Merkmalskombinationen für die drei Kriterien, das semantische, das lexikalische und das morphophonologische zusammen mit möglichen Beispielbelegungen. Insgesamt ergibt sich somit ein Spektrum von den Einheiten, die in jeder der berücksichtigten drei Hinsichten Suffixe sind, bis hin zu jenen Einheiten, die in diesen Hinsichten eindeutig Kompositionsglieder sind.

Deutlich geworden dürfte sein, dass auch bei diesem differenzierten Verfahren der Bereich diskret gegliedert wird. Das Konzept eines möglichen „Kontinuums“ zwischen Affigierung und Komposition wird somit nicht abgebildet - dies ist ja im strikten Sinne nie möglich, solange eine (beliebig große) Menge von binären Merkmalen benutzt wird. 


\begin{tabular}{|c|c|c|c|c|c|c|c|c|}
\hline & Typ 1 & Typ 2 & Typ 3 & Typ 4 & Typ 5 & Typ 6 & Typ 7 & Typ 8 \\
\hline $\begin{array}{l}\text { Seman- } \\
\text { tisches } \\
\text { Kriterium }\end{array}$ & $\begin{array}{c}{[+} \\
\text { Suffix] }\end{array}$ & $\begin{array}{c}{[+} \\
\text { Suffix] }\end{array}$ & $\begin{array}{c}{[+} \\
\text { Suffix }]\end{array}$ & $\begin{array}{c}{[+} \\
\text { Suffix }]\end{array}$ & $\underset{\text { Suffix }]}{[-}$ & $\stackrel{[-}{{ }_{\text {Suffix }}}$ & $\stackrel{[-}{\text { Suffix }]}$ & $\stackrel{[-}{[-}$ \\
\hline $\begin{array}{l}\text { Lexika- } \\
\text { lisches } \\
\text { Kriterium }\end{array}$ & $\begin{array}{c}{[+} \\
\text { Suffix] }\end{array}$ & $\begin{array}{c}{[+} \\
\text { Suffix] }\end{array}$ & $\stackrel{[-}{\text { Suffix }]}$ & $\stackrel{[-}{\text { Suffix }]}$ & $\begin{array}{c}{ }^{+} \\
\text {Suffix] }\end{array}$ & $\begin{array}{c}{ }^{+} \\
\text {Suffix] }\end{array}$ & $\stackrel{[-}{\text { Suffix }^{-}}$ & $\stackrel{[-}{\text { Suffix] }}$ \\
\hline \multirow[t]{2}{*}{$\begin{array}{l}\text { Morpho- } \\
\text { phono- } \\
\text { logisches } \\
\text { Kriterium } \\
\end{array}$} & $\begin{array}{c}{[+} \\
\text { Suffix }]\end{array}$ & $\stackrel{[-}{\text { Suffix] }}$ & $\begin{array}{c}{ }^{+} \\
\text {Suffix] }\end{array}$ & $\stackrel{[-}{\text { Suffix }^{-}}$ & 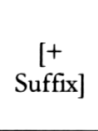 & $\stackrel{[-}{\text { Suffix }^{-}}$ & $\begin{array}{c}{[+} \\
\text { Suffix }]\end{array}$ & $\stackrel{[-}{[-}$ \\
\hline & $-n i s$ & -schaft & $\varnothing$ & $-g u t$ & ø & $\begin{array}{l}\text { Kon- } \\
\text { fix:- } \\
\text { therm }\end{array}$ & ø & $\begin{array}{l}\text { Kompo- } \\
\text { sitions- } \\
\text { glied }\end{array}$ \\
\hline \multicolumn{9}{|c|}{ Termini $1 \underbrace{\underbrace{}_{\text {,Halbsuffix }}}_{\text {,Suffix }}$} \\
\hline Termini 2 & ,Suffix & & albsuff & & & & & \\
\hline Termini 3 & ,Suffix & & & & $\mathrm{ffix}^{6}$ & & & \\
\hline
\end{tabular}

Die Tabelle zeigt, dass von den 8 kombinatorischen Möglichkeiten drei ausgeschlossen sind, da die Merkmale keineswegs völlig voneinander unabhängig sind. Ausgeschlossen sind:

- Einheiten mit grammatischer Bedeutung, die ein lexikalisches Homonym haben und morphophonologisch Suffixstatus haben (Typ 3)

- Einheiten mit lexikalischer Bedeutung, die kein lexikalisches Homonym haben und morphophonologisch Suffixstatus haben (Typ 5)

- Einheiten mit lexikalischer Bedeutung, die ein lexikalisches Homonym haben und morphophonologisch Suffixstatus haben (Typ 7)

Es ergibt sich ferner, dass auch die so genannten ,Konfixe‘, Einheiten, die nur gebunden vorkommen, aber eine lexikalisch ausdifferenzierte Bedeutung haben wie (-)therm(-), (-)naut(-), (-) log(-) in Thermik, exotherm; nautisch, Astronaut; Logistik, Biologie, analog, hier als Typ 6 eingereiht werden können. 
Ebenfalls eingetragen sind die beiden erwähnten terminologischen Alternativen. Die Alternative 1 lässt sich - vgl. die Argumentation oben - vom Primat des lexikalischen Kriteriums leiten und fasst Einheiten wie -nis, -ling usw. auf der eine Seite und -schaft, -tum auf der anderen Seite als Suffixe zusammen. Die Alternative 2 hingegen setzt das morphophonologische Kriterum primär und fasst letztere mit Einheiten wie -gut, -fall, -werk, -zeug zusammen als Halbaffixe. Letztlich ist auch die terminologische Alternative 3 zu nennen, bei der alle Elemente zwischen den beiden Polen, also die Typen 2 bis 7 als Halbaffixe bezeichnet werden. Hier liegt dann jene Verwendung als allgemeine Sammelbezeichnung vor, die keine Kategorie bezeichnet, deren Elemente alle eine gemeinsame Schnittmenge an Merkmalen aufwiesen.

\section{Resümee}

1. Singulativ- und Kollektivbildungen können als zwei zueinander komplementäre Bildungsweisen zur Erzeugung von Substantiven mit einer bestimmten Seinsart bzw. einem bestimmten Nominalaspekt, jeweils dem individuativen und dem kontinuativen Nominalaspekt, betrachtet werden.

2. Singulativbildung ist im Rahmen der deutschen Wortbildung nur schwach grammatikalisiert. Ansätze zur Grammatikalisierung zeigen nur die Singulativmarker -fall und -stück. Kollektivbildung ist dagegen durch Affixe wie ge-, -schaft usw. fest etabliert. Die auf Kompositionsgliedern beruhenden -gut, -werk und -zeug ergänzen das derivationelle Subsystem. Sie sind vergleichsweise stärker grammatikalisiert.

3. Indizien für Grammatikalisierung sind u.a. Abweichungen in der ,Seinsart ${ }^{\star}$ gegenüber selbstständiger Verwendung, Lizensierung verschiedener Sorten von Erstgliedern und die Unzulässigkeit des selbstständigen Vorkommens in appositiven Strukturen bzw. Numerativkonstruktionen. Die Kompositionsfreudigkeit des Deutschen kommt hier einem grammatischen Zweck zugute, dem (ansatzweisen) Ausdruck von Nominalaspekt.

4. Die Einordnung der bereits grammatikalisierten Singulativ- und Kollektivmarker als Halbaffixe/Affixoide ist kritisch zu prüfen. Es sind mindestens drei Kriterien in Rechnung zu stellen, das lexikalische, das semantische und das morphophonologische, die jeweils in Richtung 
Kompositionsglied bzw. Affix deuten. Von den acht kombinatorisch möglichen Typen, die den Raum vom reinen Suffix bis zum reinen Kompositionsglied ausmessen, sind im Deutschen fünf vertreten. Drei davon, unter ihnen die hier behandelten Singulativ- und Kollektivmarker, könnten unter die Sammelbezeichnung Halbaffix fallen.

\section{Literatur}

Birkenmaier, Willy (1980): „Die Kategorie ,zählbar - nichtzählbar ‘ beim russischen Substantiv". International Review of Applied Linguistics in Language Teaching 18/1, 3140.

Booij, Geert (2009): „Compounding and construction morphology“. In: Rochelle Lieber \& Pavol Štekauer (Hg.), The Oxford Handbook of Compounding. Oxford: University Press, 201-16.

Bunt, Harry C. (1985): Mass Terms and Model-Theoretic Semantics. Cambridge u.a.: Cambridge University Press.

Drossard, Werner (1982): „Die Technik MASSE/MESSEN auf der Dimension der Apprehension". In: Hansjakob Seiler \& Christian Lehmann (Hg.), Apprehension. Das spracbliche Erfassen von Gegenständen. Teil I: Bereich und Ordnung der Phänomene. Tübingen: Narr. 98-111.

Duden (2005): Die Grammatik. Bd.7. völlig neu erarbeitete und erweiterte Auflage. Mannheim u.a.: Duden.

DWB: Deutsches Wörterbuch. Elektronische Ausgabe der Erstbearbeitung von Jakob und Wilhelm Grimm (,Der digitale Grimm“). Frankfurt am Main: Zweitausendeins.

Eisenberg Peter (2004): Grundriß der deutschen Grammatik. Band 1: Das Wort. 2. Aufl. Stuttgart \& Weimar: Metzler.

Fleischer, Wolfgang (1983): Wortbildung der deutschen Gegenwartssprache. Leipzig: VEB.

Fleischer, Wolfgang \& Irmhild Barz (1995): Wortbildung der deutschen Gegenwartssprache. 2. Aufl. Tübingen: Niemeyer.

Greenberg, Joseph (1971): „Numeral classifiers and substantival number. Problems in the genesis of a linguistic type“. In: Luigi Heilmann (Hg.), Proceedings of the $11^{\text {th }} \mathrm{In}$ ternational Congress of Linguistics I. Bologna: Il Mulino. 17-37.

Hopper, Paul J. \& Elizabeth Closs Traugott (2003): Grammaticalization. 2nd ed. Cambridge: University Press.

Jackendoff, Ray (1991): „Parts and boundaries“. Cognition 41, 9-45.

Kastovsky, Dieter (2009): „Diachronic perspectives“. In: Rochelle Lieber \& Pavol Štekauer (Hg.), The Oxford Handbook of Compounding. Oxford: University Press. 323-40.

Koptjevskaja-Tamm, Maria (2004): „Mass and Collection“. In: Geert Booij u.a. (Hg.), Morphologie. Ein internationales Handbuch zur Flexion und Wortbildung. 2. Halbband. Berlin \& New York: De Gruyter, 1067-73 (= Handbücher zur Sprach- und Kommunikationswissenschaft 17/2). 
Krifka, Manfred (1991): „Massennomina“. In: Arnim von Stechow \& Dieter Wunderlich (Hg.), Semantik. Ein internationales Handbuch der zeitgenössischen Forschung. Berlin \& New York: De Gruyter, 399-417 (= Handbücher zur Sprach- und Kommunikationswissenschaft 6).

Kuhn, Wilfried (1982): „Kollektiva und Technik KOLLEKTION am Beispiel des Deutschen“. In: Hansjakob Seiler \& Christian Lehmann (Hg.), Apprehension. Das sprachliche Erfassen von Gegenständen. Teil I: Bereich und Ordnung der Pbänomene. Tübingen: Narr. 84-97.

Löbel, Elisabeth (1986): Apposition und Komposition in der Quantifizierung. Syntaktische, semantische und morphologische Aspekte quantifizierender Nomina im Deutschen. Tübingen: Niemeyer (=Linguistische Arbeiten 166).

Motsch, Wolfgang (2004): Die deutsche Wortbildung in Grundzügen. 2. überarb. Aufl. Berlin \& New York: De Gruyter (= Schriften des Instituts für Deutsche Sprache 8).

Ortner, Lorelies et al. (1991): Deutsche Wortbildung. Typen und Tendenzen in der Gegenwartssprache. Eine Bestandsaufnabme des Instituts für deutsche Sprache Forscbungsstelle Innsbruck. Vierter Hauptteil. Substantivkomposita. Komposita und kompositionsähnliche Strukturen. Berlin \& New York: De Gruyter (= Sprache der Gegenwart LXXIX).

Raffelsiefen, Renate (demn.): Wortinterne Domänen im Deutschen und Englischen. Erscheint in der Reihe amades (Arbeitspapiere und Materialien zur deutschen Sprache) des Instituts für Deutsche Sprache.

Rijkhoff, Jan (2004): The Noun Phrase. 2. Aufl. Oxford: Oxford University Press.

Thieroff, Rolf (2000): Morphosyntax nominaler Einheiten im Deutschen. Manuskript Bonn.

Van Goethem, Kristel (2008): „Oud-leerling versus ancien élève: A Comparative Study of Adjectives Grammaticalizing into Prefixes in Dutch and French". International Journal of Morphology 18/1, 27-49.

Vendler, Zeno (1957): „Verbs and Times“. The Philosophical Review 66/2, 143-60.

Wellmann, Hans (1969): Kollektiva und Sammelwörter im Deutschen. Dissertation Universität Bonn.

Wellmann, Hans (1975): Das Substantiv. Deutsche Wortbildung. Zweiter Hauptteil. Düsseldorf: Schwann.

Wiese, Heike (1997): Zabl und Numerale. Eine Untersuchung zur Korrelation konzeptueller und sprachlicher Strukturen. Berlin: Akademie (= Studia grammatica 44).

Zifonun, Gisela (2009): „Wie wir zu -FALL kommen“. Sprachreport 2/2009, 2-3.

Zifonun, Gisela (2010): „Possessive Attribute im Deutschen“. In: Lutz Gunkel \& Jan Rijkhoff (Hg.): Deutsche Sprache 02/2010. Themenheft: Modifikation im Deutschen: Kontrastive Untersuchungen zur Nominalphrase, 124-53. 